\title{
First-Principles Simulations of Lithium Melting: Stability of the bec Phase Close to Melting
}

\author{
E. R. Hernández, ${ }^{1,2, *}$ A. Rodriguez-Prieto, ${ }^{3,4}$ A. Bergara, ${ }^{5,6}$ and D. Alfè $\mathrm{e}^{3,4,7, \dagger}$ \\ ${ }^{1}$ Institut de Ciència de Materials de Barcelona (ICMAB-CSIC), Campus de Bellaterra, 08193 Barcelona, Spain \\ ${ }^{2}$ Instituto de Ciencia de Materiales de Madrid (ICMM-CSIC), Campus de Cantoblanco, 28047 Madrid, Spain \\ ${ }^{3}$ Department of Physics and Astronomy, University College London, London WC1E 6BT, United Kingdom \\ ${ }^{4}$ Thomas Young Centre, University College London and London Centre for Nanotechnology, University College London, \\ London WC1E 6BT, United Kingdom \\ ${ }^{5}$ Materia Kondesatuaren Fisika Saila, UPV/EHU, 644, E-48080 Bilbo, Spain \\ ${ }^{6}$ DIPC and Centro de Física de Materiales CSIC-UPV/EHU, E20080 Donostia, Spain \\ ${ }^{7}$ Department of Earth Sciences, University College London, London WC1E 6BT, United Kingdom \\ (Received 5 October 2009; revised manuscript received 15 February 2010; published 3 May 2010)
}

\begin{abstract}
We report large-scale first-principles simulations of melting of four different phases of Li at pressures ranging from 0 to $50 \mathrm{GPa}$. We find excellent agreement with existing experimental data at low pressures, and confirm that above $10 \mathrm{GPa}$ the melting line develops a negative slope, in parallel to what occurs for $\mathrm{Na}$ at $30 \mathrm{GPa}$. Surprisingly, our results indicate that the melting temperature of the bcc phase is always higher than that of fcc Li, suggesting the intriguing possibility of the existence of a narrow field of bcc stability separating the fcc and liquid phases, as predicted by Alexander and McTague [Phys. Rev. Lett. 41, 702 (1978)].
\end{abstract}

DOI: 10.1103/PhysRevLett.104.185701

The alkali elements $\mathrm{Li}$ and $\mathrm{Na}$ have long been described as simple metals, due to their prototypical atomic and electronic structure. At ambient conditions they adopt the bcc lattice arrangement, transforming into the fcc at higher pressure (at $\sim 7.5 \mathrm{GPa}$ and room temperature in the case of $\mathrm{Li}$, at $65 \mathrm{GPa}$ in the case of $\mathrm{Na}$ ), while their electronic structure is nearly free electronlike. However, recent theoretical and experimental studies have shown that these materials display unexpectedly complex behavior at high pressures. Neaton and Ashcroft [1,2] reported ab initio calculations predicting that pressure induces phase transitions to less symmetric, lower coordinated structures, associated to electronic localization [3,4], a rather counterintuitive prediction. Shortly after, Hanfland et al. [5] reported an experimental study of Li at high pressures, and although the actual sequence of high-pressure phases found by these authors was different than that predicted theoretically [1], they nevertheless confirmed the tendency to adopt low symmetry phases at high pressures. Other experimental efforts have shown that these high-pressure structural transformations are accompanied by changes in the electronic structure. Indeed, Struzhkin et al. [6] and Shimizu and coworkers [7] have reported the observation of superconductivity in $\mathrm{Li}$ at pressures above $20 \mathrm{GPa}$ (at ambient pressure $T_{c}=0.4 \mathrm{mK}$ [8]), with transition temperatures varying with pressure, but ranging between 9 and up to $20 \mathrm{~K}$, one of the highest measured for any element. Matsuoka and Shimizu [9] have reported a metal-tosemiconductor transition in Li near $80 \mathrm{GPa}$ of pressure. Similar results have been reported for $\mathrm{Na}$ at $118 \mathrm{GPa}$, where $\mathrm{Na}$ is found to transform to an orthorhombic phase behaving as a poor metal [10]. At even higher pressures (c.a. $200 \mathrm{GPa}$ ) Ma and coworkers [11] have reported the
PACS numbers: 64.70.dj, 62.50.- $\mathrm{p}, 65.20 .-\mathrm{w}, 65.40 .-\mathrm{b}$

observation of a sixfold coordinated hexagonal structure of $\mathrm{Na}$ which happens to be transparent, implying an insulator band gap in the electronic structure.

In an effort to further characterize the thermodynamic behavior of alkali metals at high pressures, Gregoryanz et al. [12] have measured the melting temperature of Na up to pressures of $130 \mathrm{GPa}$. It was found that its melting curve reaches a maximum at $\sim 31 \mathrm{GPa}$, and decreases at higher pressures. The negative slope in the melting line persists up to pressures of $\sim 118 \mathrm{GPa}$, conditions at which $\mathrm{Na}$ is found to be in the liquid state at room temperature, and where a number of highly complex crystalline phases are found in the solid [13], one of which has been recently proved to be incommensurate [14]. While a negative slope in the melting line is itself not unprecedented, and in fact is known to occur in many systems (notably, water), it usually occurs only over a narrow pressure range. A negative slope persisting over more than $70 \mathrm{GPa}$, as occurs in $\mathrm{Na}$, is quite without precedent. To date, no experimental measurements of the melting curve of $\mathrm{Li}$ at pressures beyond $8 \mathrm{GPa}$ have been reported, and given the unusual melting behavior of $\mathrm{Na}$, and the no less remarkable one of $\mathrm{K}$, there is considerable interest in the melting of $\mathrm{Li}$ at pressures higher than $8 \mathrm{GPa}$.

In this work we use first-principles molecular dynamics (FPMD) simulations of $\mathrm{Li}$ at pressures ranging between 0 and $50 \mathrm{GPa}$, using this technique to determine the melting temperature of four different crystalline phases found within this pressure range.

There are several possibilities for calculating the melting temperature of a given model material. One approach consists of calculating the free energy of the solid and liquid phases at fixed pressure as a function of the tem- 
perature; the temperature at which the free energies of both phases are equal is the sought coexistence temperature. This approach has been used successfully in numerous applications [15-17]; it is conceptually simple, and besides the coexistence conditions, it provides access to a wealth of thermodynamic data, such as the enthalpy of fusion, entropy, volume, etc. But the free energies are cumbersome to calculate, and have to be evaluated with high precision, especially in cases where the entropy difference between solid and liquid is small (the free energies cross at a small angle) to avoid large errors in the coexistence temperature. A second approach, which is in fact the one used in this work, consists of considering simultaneously solid and liquid within the same simulation box. If the simulation is performed at fixed temperature the thermodynamically unstable phase disappears, while the stable one prevails, and it is thus possible to bracket the coexistence temperature by repeating the same simulation at different temperatures to within a small temperature interval. Alternatively, one can manually adjust the enthalpy (by temperature scaling) until both phases are observed to remain in coexistence for a sufficiently long time, without any appreciable trend towards melting or solidification. This method, though extensively used with empirical potentials, was not used in combination with first-principles simulations until very recently $[18,19]$ due to the requirement of using large supercells. A third method, which we call the direct method, has been used occasionally. This consists of simulating the solid phase at increasing temperatures, until transition to the liquid is finally observed. This method provides the so-called mechanical melting temperature, which generally overestimates the equilibrium melting temperature. It has been argued that this superheating effect should be small in the light alkali metals Li and $\mathrm{Na}[20,21]$, but further work is needed to establish the reliability of this approach in these systems.

We performed FPMD simulations of Li systems in the isobaric ensemble (constant number of particles, $\mathrm{N}$, constant-pressure, $\mathrm{P}$, and constant enthalpy, H). Simulations were performed using the Vienna $a b$ initio Simulation Package (VASP) [22]. We employed the projector augmented wave method [23] as implemented in VASP [24], retaining only the $2 \mathrm{~s}$ electron in the valence, and the exchange-correlation energy was described employing the functional due to Perdew, Burke and Ernzerhof [25]. We used a plane-wave cutoff of $10.3 \mathrm{Ry}$, and the Brillouin zone was sampled only with the $\Gamma$ point. The FPMD simulations employed an efficient charge density extrapolation scheme [26] which speeds up the attainment of self-consistency after the first few dynamical steps. Isobaric ensemble trajectories for the ions and cell were generated employing the constant-pressure formalism of Souza and Martins [27] as implemented in [28], using a time step of $3 \mathrm{fs}$. The simulation box was constrained to remain in a tetragonal shape, with the two short sides (parallel to the plane of the interface) being of equal length. Coexistence simulations require large simulation cells in order to have a sufficient number of atoms in each (solid or liquid) phase. The number of atoms was 2048 atoms in simulations involving the bcc or cI16 phases, 2016 atoms in those involving the fcc phase, and 2058 atoms for those involving the hR1 phase. The preparation of initial configurations for these simulations was done employing a simple adjusted embedded atom potential, fitted to reproduce the results obtained from small $a b$ initio simulations at the conditions of interest. Initial configurations containing approximately equal amounts of solid and liquid plus an interface between them were straight forwardly generated with the empirical potential, and then fed to the $a b$ initio coexistence simulation.

By monitoring the behavior of the instantaneous temperature during such simulations we were able to determine if the solid and liquid phase were in coexistence or not. A tendency of the instantaneous temperature to grow (decrease) during the course of the simulation is indicative of a tendency of the whole system to solidify (melt). On the other hand, the absence of any appreciable drift in the instantaneous temperature is indicative of the proximity to coexistence conditions. Thus, when a drift was found the enthalpy was manually adjusted by rescaling the temperature of the simulation so as to drive the system towards coexistence. In the absence of any detectable temperature drift, and when both phases were found to coexist stably for a minimum of $15 \mathrm{ps}$, they were assumed to be in equilibrium at the average temperature and pressure conditions of the simulation. We find that the standard deviation of the temperature in such conditions (which we take as an estimate of the error in our coexistence temperatures) is smaller than $10 \mathrm{~K}$; the standard deviation in the pressure is smaller than $0.1 \mathrm{GPa}$. It is our experience, both in the present work and from previous research [18], that the stable coexistence of solid and liquid phases is extremely sensitive to the temperature, and the small standard deviation of the coexistence temperatures resulting from our simulations corroborate this observation. The presence of both solid and liquid phases within the simulation box can be easily corroborated either by direct inspection or by plotting the particle density calculated at a series of planes parallel to the interface [see Fig. 1]. Crystal planes appear as spikes of high density, whereas no such spikes are present in the liquid region.

In this work we have considered the melting of four different crystalline phases of Li, namely, the bcc, fcc, hR1, and cI16 phases, which are the relevant phases from ambient pressure up to $50 \mathrm{GPa}$, the range of pressures that we have spanned in this study. Phase hR1 (labeled here by its Pearson symbol) is rhombohedral with space group $R \overline{3} \mathrm{~m}$. Phase cIl6 is cubic, with space group $I \overline{4} 3 d$, and has 16 nonequivalent atoms in the unit cell.

Figure 2 displays the melting temperatures that resulted from our simulations. Estimated errors in the calculated melting temperatures are comparable to the symbol sizes. Also shown are the experimental data Ludemann and Kennedy [29] (in the range 0.5 to $8 \mathrm{GPa}$ ) and Boehler 


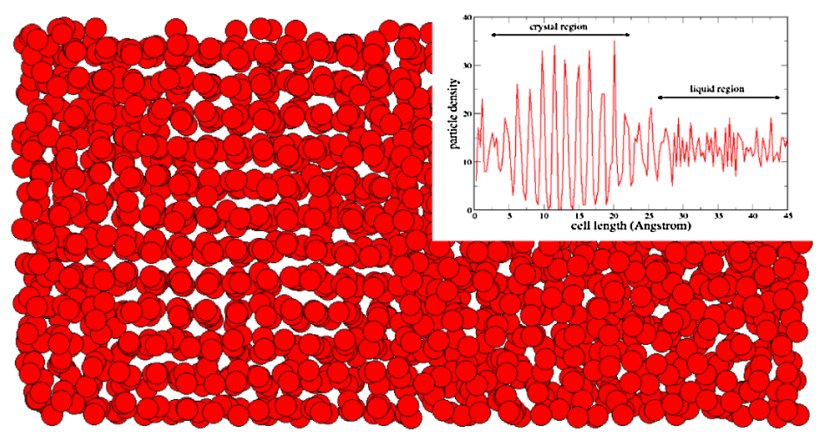

FIG. 1 (color online). Instantaneous configuration resulting from a coexistence simulation of the fcc phase and liquid $\mathrm{Li}$ at $30 \mathrm{GPa}$. Crystal planes are clearly visible on the left side of the image, and the interfaces between solid and liquid are discernible at the center of the image and the left end. The inset shows the particle density of the configuration shown in the main image. Regularly spaced density peaks reveal the presence of crystal planes on the left, whereas the small density oscillations around a constant value are typical of the liquid phase, on the right of the image.

[30] (in the range 0 to $3 \mathrm{GPa}$ ) for the melting of the bcc phase up to $8 \mathrm{GPa}$, the only existing experimental data on melting of Lithium that we are aware of. As can be seen in Fig. 2, over the range of pressures where experimental data is available our calculated results are in rather good agreement with the experimental melting temperatures. For example, Boehler [30] reports a melting temperature at $0 \mathrm{GPa}$ of $453 \mathrm{~K}$, while we obtain $455 \mathrm{~K}$. At $4 \mathrm{GPa}$ Ludemann and Kennedy [29] obtained a value of $516 \mathrm{~K}$, while we get $513 \mathrm{~K}$, and at $8 \mathrm{GPa}$ they report a value of $527 \mathrm{~K}$, compared to our own value of $528 \mathrm{~K}$. This rather remarkably good agreement between the available experimental data and our results for the bcc phase gives us confidence in the reliability of the approach followed

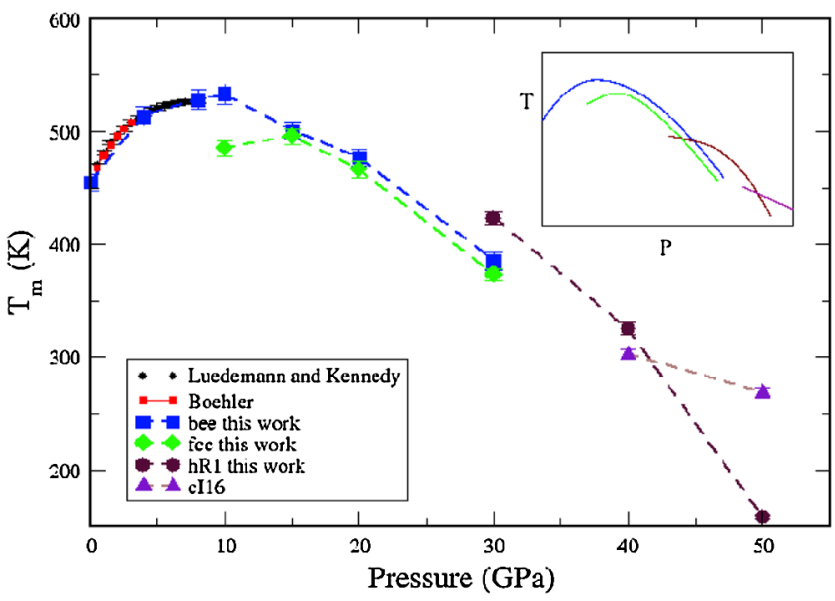

FIG. 2 (color online). Calculated melting temperatures for the bcc, fcc, hR1, and cI16 phases. Also plotted is the experimental data of Ludemann and Kennedy [29] and Boehler [30]. The inset shows a schematic diagram of the melting line up to $50 \mathrm{GPa}$, as envisaged from our numerical results. here, both for the bcc structure and the remaining phases considered in this study.

Since the bcc phase transforms to the fcc structure at approximately $7.5 \mathrm{GPa}$ [31-33] at $300 \mathrm{~K}$, and the latter phase remains stable up to $38 \mathrm{GPa}$ [5], it is perhaps natural to assume that within this range of pressures Li should melt from the fcc phase. However, our results indicate that the bcc structure has a higher melting temperature than the fcc at all pressures up to $30 \mathrm{GPa}$, the highest pressure at which we have considered these phases. The difference in melting temperatures is not large (e.g., $\sim 5 \mathrm{~K}$ at $15 \mathrm{GPa}, \sim 10 \mathrm{~K}$ at $20 \mathrm{GPa}$ ), but nevertheless we systematically find a higher melting temperature for the bcc phase than for the fcc. This small difference is indicative of very similar free energy values for both structures at temperatures close to melting. This finding also unveils an intriguing possibility: that a narrow stability field exists for the bcc phase between the fcc phase and the liquid. It would be very interesting to experimentally check this possibility, which seems all the more likely in view of the work of Alexander and McTague [34]. Using symmetry arguments and a mean-field description of the solid-liquid transition, these authors proved that, within the range of applicability of mean-field theory, the melting temperature of the bcc structure should be higher than that of any other lattice arrangement, and, in particular, higher than that of the fcc structure. Their argument is supported by the fact most metallic elements, lanthanides and actinides adopt the bcc structure close to the melting line.

Another remarkable observation to be extracted from the results displayed in Fig. 2 is the fact that, within the range of pressures spanned here, the maximum melting temperature is obtained in the region from 8 to $10 \mathrm{GPa}$, where the bcc phase melts at $\sim 530 \mathrm{~K}$, which is considerably lower than the highest mechanical melting temperature obtained by Tamblyn et al. [21] (680 K) in their direct method simulations. At higher pressures both the bcc and fcc phases melt at lower temperatures, i.e., the melting line has a negative slope. In this respect Li behaves similarly to $\mathrm{Na}$, where it has been shown experimentally [12] that the melting line of the bcc phase attains a maximum at $\sim 31 \mathrm{GPa}$ with a value close to $1000 \mathrm{~K}$, and then decreases reaching values close to room temperature at about $100 \mathrm{GPa}$. Several studies have attempted to uncover the cause of the negative slope in the melting line of $\mathrm{Na}$, some arguing for the higher compressibility of the liquid as the explanation [20,35], while others have drawn attention to a softening of phonon modes with increasing pressure, resulting in a relative destabilization of the solid phase [3638]. We note that these explanations are not mutually exclusive, and it is likely that both contribute to the observed negative slope in the melting line of sodium.

At $30 \mathrm{GPa}$ the melting temperature of the $\mathrm{hR} 1$ phase is some $35 \mathrm{~K}$ higher than that of the bcc phase (40 K higher than that of fcc), which is indicative of the higher stability of the rhombohedral phase. According to Hanfland et al. [5], the hR1 phase appears at $\sim 38 \mathrm{GPa}$ at $180 \mathrm{~K}$, which is 
reasonably consistent with our finding of this phase being the most stable one at $30 \mathrm{GPa}$ and $T=425 \mathrm{~K}$. This phase also presents a negative slope of the melting line, as its melting temperature reduces to $325 \mathrm{~K}$ when the pressure is raised to $40 \mathrm{GPa}$.

Finally, we have considered the melting of the cI16 phase at 40 and $50 \mathrm{GPa}$, known [5] to be the stable solid phase in this pressure range. We find that this phase melts at $302.5 \mathrm{~K}$ at $40 \mathrm{GPa}$, and at $272 \mathrm{~K}$ at $50 \mathrm{GPa}$, thus continuing the trend towards lower melting temperatures as the pressure is increased already found in the bcc, fcc, and hR1 phases.

In summary, we have carried out large-scale firstprinciples simulations of solid-liquid coexistence of different Li crystal phases. From these simulations we have obtained the melting temperature of these phases, covering a range of pressures from 0 to $50 \mathrm{GPa}$. Our calculated melting temperatures for the bcc phase agree very closely with the available experimental data existing for this phase [29,30], between 0 and $8 \mathrm{GPa}$. The highest melting temperature in the range of pressures considered is observed for the bcc phase, occurring at $10 \mathrm{GPa}(533 \mathrm{~K})$; at higher pressures the melting lines of bcc and fcc phases develop a negative slope, i.e., the melting temperature reduces with pressure, in agreement with the earlier find of Tamblyn et al. [21], and this trend is maintained by the hR1 and cI16 phases. This predicted behavior is similar to that found experimentally in the case of $\mathrm{Na}$ [12]. We also find that up to $30 \mathrm{GPa}$, the melting temperature of the bcc phase is systematically higher than that of the fcc phase, in spite of the fact that at low temperatures it is clearly established that the latter phase is the stable one above $\sim 8 \mathrm{GPa}$. This implies that, according to our calculations, there must exist a narrow field of bec stability separating the fcc and liquid fields.

The authors gratefully acknowledge the use of computer resources and technical assistance provided by the Barcelona Supercomputing Centre (BSC); E. R.H. and D. A. also thank the Royal Society for funding this work. The work of E. R. H. is supported by the Spanish Ministry of Science and Innovation through Project No. FIS200612117-C04-01; A. B. thanks GV-UPV/EHU for Project No. IT-366-07. The work of D. A. was conducted as part of an EURYI scheme grant as provided by EPSRC-GB.

Note added in proof.-Recently we became aware of the work by Lazicki et al. [39], who extend the experimental melting line of $\mathrm{Li}$ up to $15 \mathrm{GPa}$. These authors find a maximum in the melting line at $\sim 10 \mathrm{GPa}$, and fail to observe any signature of bcc-fcc transition along it up to $15 \mathrm{GPa}$. Like with the earlier experimental work [29,30], our results are in rather good agreement with the data reported in this recent study.

*ehe@icmm.csic.es $\dagger$ d.alfe@ucl.ac.uk

[1] J. B. Neaton and N.W. Ashcroft, Nature (London) 400, 141 (1999).

[2] J. B. Neaton and N. W. Ashcroft, Phys. Rev. Lett. 86, 2830 (2001).

[3] A. Bergara, J. B. Neaton, and N. W. Ashcroft, Phys. Rev. B 62, 8494 (2000).

[4] A. Rodriguez-Prieto and A. Bergara, Phys. Rev. B 72, 125406 (2005).

[5] M. Hanfland et al., Nature (London) 408, 174 (2000).

[6] V. V. Struzhkin et al., Science 298, 1213 (2002).

[7] K. Shimizu et al., Nature (London) 419, 597 (2002).

[8] J. Tuoriniemi et al., Nature (London) 447, 187 (2007).

[9] M. Matusuoka and K. Shimizu, Nature (London) 458, 186 (2009).

[10] A. Lazicki et al., Proc. Natl. Acad. Sci. U.S.A. 106, 6525 (2009).

[11] Y. Ma et al., Nature (London) 458, 182 (2009).

[12] E. Gregoryanz et al., Phys. Rev. Lett. 94, 185502 (2005).

[13] E. Gregoryanz et al., Science 320, 1054 (2008).

[14] L. F. Lundegaard et al., Phys. Rev. B 79, 064105 (2009).

[15] O. Sugino and R. Car, Phys. Rev. Lett. 74, 1823 (1995).

[16] D. Alfè, M. J. Gillan, and G. D. Price, Nature (London) 401, 462 (1999).

[17] M. Kaczmarski, O. N. Bedoya-Martínez, and E. R. Hernández, Phys. Rev. Lett. 94, 095701 (2005).

[18] D. Alfè, Phys. Rev. B 68, 064423 (2003).

[19] S. A. Bonev et al., Nature (London) 431, 669 (2004).

[20] J. Y. Raty, E. Schwegler, and S. A. Bonev, Nature (London) 449, 448 (2007).

[21] I. Tamblyn, J. Y. Raty, and S. A. Bonev, Phys. Rev. Lett. 101, 075703 (2008).

[22] G. Kresse and J. Furthmüller, Phys. Rev. B 54, 11169 (1996).

[23] P. E. Blöchl, Phys. Rev. B 50, 17953 (1994).

[24] G. Kresse and D. Joubert, Phys. Rev. B 59, 1758 (1999).

[25] J. P. Perdew, K. Burke, and M. Ernzerhof, Phys. Rev. Lett. 77, 3865 (1996).

[26] D. Alfè, Comput. Phys. Commun. 118, 31 (1999).

[27] I. Souza and J. L. Martins, Phys. Rev. B 55, 8733 (1997).

[28] E. Hernández, J. Chem. Phys. 115, 10282 (2001).

[29] H. D. Luedemann and G. C. Kennedy, J. Geophys. Res. 73, 2795 (1968)

[30] R. Boehler, Phys. Rev. B 27, 6754 (1983).

[31] B. Olinger and W. Shaner, Science 219, 1071 (1983).

[32] M. Hanfland et al., Solid State Commun. 112, 123 (1999).

[33] V. V. Struzhkin, R. J. Hemley, and H. K. Mao, Bull. Am. Phys. Soc. 44, 1489 (1999).

[34] S. Alexander and J. McTague, Phys. Rev. Lett. 41, 702 (1978).

[35] E. R. Hernández and J. Íñiguez, Phys. Rev. Lett. 98, 055501 (2007).

[36] M. Martinez-Canales and A. Bergara, J. Phys. Chem. Solids 69, 2151 (2008).

[37] L. Koci, R. Ahuja, L. Vitos, and U. Pinsook, Phys. Rev. B 77, 132101 (2008).

[38] S. V. Lepeshkin, M. V. Magnitskaya, and E. G. Maksimov, JETP Lett. 89, 586 (2009).

[39] A. Lazicki et al., Solid State Commun. 150, 625 (2010). 